\title{
Integrated open surgical and endovascular embolization treatment of a paracavernous venous plexus fistula: case report
}

\author{
Justin A. Dye, MD, Colin C. Buchanan, MD, and Nestor R. Gonzalez, MD \\ Department of Neurosurgery, David Geffen School of Medicine, University of California, Los Angeles, California
}

The authors report the treatment of a rare type of dural arteriovenous fistula of the paracavernous venous plexus. These fistulas can mimic carotid-cavernous fistulas in both imaging characteristics and clinical presentation, but the anatomical differences require differences in management. The authors describe an integrated open surgical and direct endovascular embolization approach and review of the literature pertaining to the anatomy of and treatment options for paracavernous fistulas.

http://thejns.org/doi/abs/10.3171/2014.11.JNS14958

KEY WORDS carotid-cavernous fistula; combined treatment; endovascular embolization; paracavernous venous plexus; vascular disorders

$\mathrm{P}$ ARACAVERNOUS venous plexus fistulas are rare types of dural arteriovenous fistulas (DAVFs) that can mimic indirect carotid-cavernous fistulas (CCFs) in both imaging characteristics and clinical presentation. While similar to CCFs, their anatomical differences lead to differences in management. Key differences include the distinct patterns of venous drainage, which affect the options for endovascular access, and the actual location of the abnormal shunt. Both types of fistula may have drainage through the superior ophthalmic vein, the plexus of the foramen ovale to the pterygoid plexus, and/or the deep middle cerebral vein. However, unlike a $\mathrm{CCF}$, the paracavernous venous plexus fistula does not drain directly through the inferior petrosal sinus, but rather it drains through the cavernous sinus. Because the fistulous connection resides in the paracavernous venous plexus and not in the cavernous sinus, embolization of the cavernous sinus does not treat the fistula and can endanger the patient by altering the drainage toward cerebral veins (Fig. 1). This anatomical difference in location and venous drainage can make these types of DAVFs very difficult to treat by endovascular interventions alone.

In this report the authors describe the presentation, imaging findings, and integrated open surgery and endovascular embolization of a rare type of DAVF, a paracavernous venous plexus fistula.

\section{Case Report}

History and Examination

A 66-year-old right-handed Asian man presented with a 2-year history of persistent right eye chemosis. He was initially evaluated by an ophthalmologist who prescribed steroid eye drops, resulting in mild temporary improvement. However, the symptoms recurred and persisted. He reported right-sided occipital region headaches occurring approximately 3 times per week and mild right orbital proptosis. At presentation, he denied eye pain, recent change in his visual acuity, double vision, photophobia, nausea, and neck stiffness. On examination, he was found to have right eye chemosis, proptosis, and an orbital bruit. His right eye intraocular pressure was measured at $19 \mathrm{~mm} \mathrm{Hg}$. His visual fields were full to confrontation. Brain MRI/ MR angiography revealed mild right proptosis without obvious prominence of the right cavernous sinus or enlargement of the right superior ophthalmic vein. Cerebral angiography demonstrated an arteriovenous shunt of the right paracavernous venous plexus (Fig. 2). The fistula was fed by meningeal branches of a dilated inferolateral trunk, the right accessory meningeal artery, and the artery of the foramen rotundum. There was early filling of the left cavernous sinus across the intercavernous sinus. The venous drainage of the fistula was predominantly through the

ABBREVIATIONS $\mathrm{CCF}=$ carotid-cavernous fistula; $\mathrm{CTA}=\mathrm{CT}$ angiography; DAVF = dural arteriovenous fistula; ICA = internal carotid artery; LCS = laterocavernous sinus; LWSS = lesser wing of the sphenoid sinus; SMCV = superficial middle cerebral vein.

SUBMITTED April 30, 2014. ACCEPTED November 6, 2014.

INCLUDE WHEN CITING Published online December 19, 2014; DOI: 10.3171/2014.11.JNS14958.

DISCLOSURE The authors report no conflict of interest concerning the materials or methods used in this study or the findings specified in this paper. 

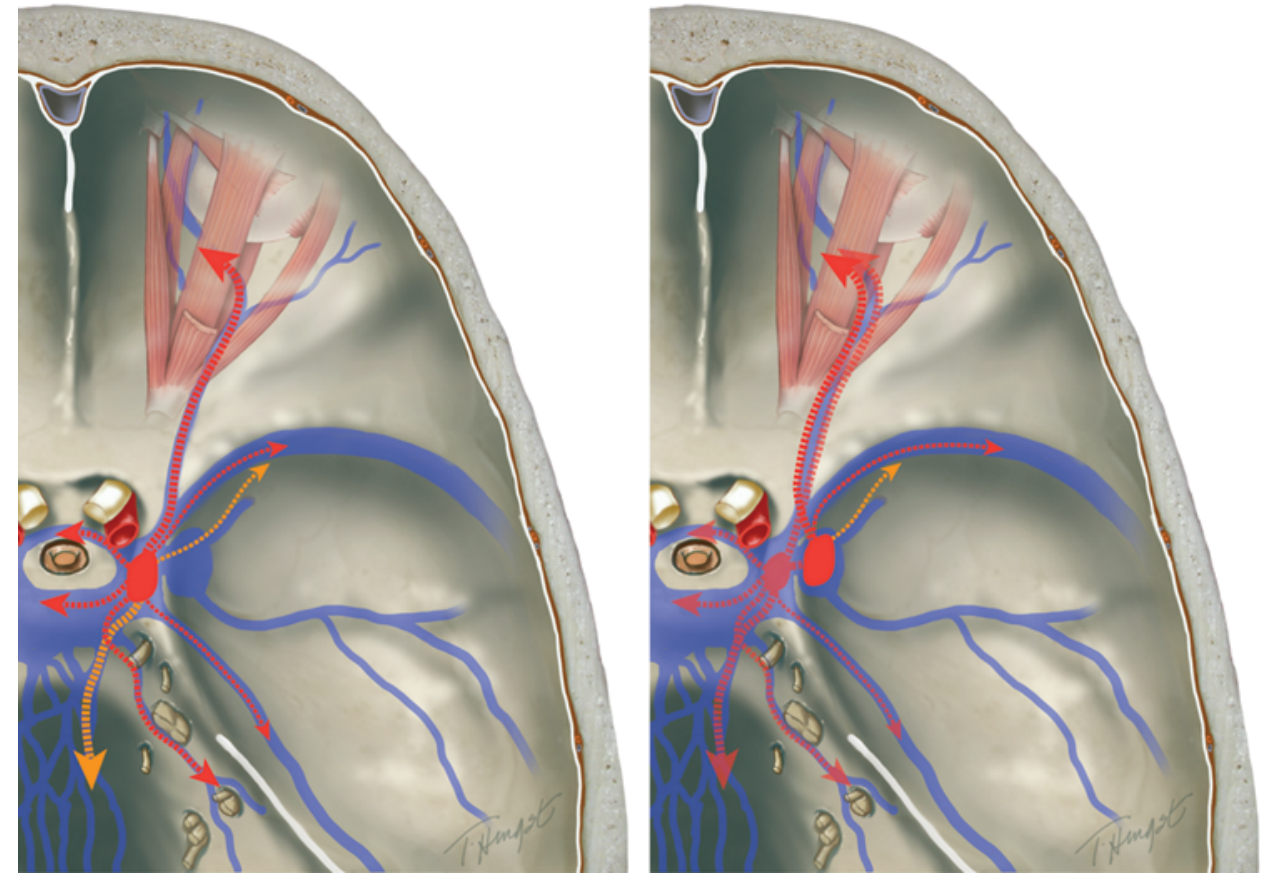

FIG. 1. The potential draining veins associated with cavernous sinus (left) versus paracavernous venous plexus (right) fistulas are demonstrated. Both share potential outlet paths through the superior ophthalmic vein (thick red arrow), the plexus of the foramen ovale to the pterygoid plexus (thin orange arrow), and the deep middle cerebral vein (thin red arrows). However, a paracavernous fistula would not have direct venous drainage through the inferior petrosal sinus, contralateral cavernous sinus (middle-size red arrows), or the perimesencephalic veins (thick orange arrow). Copyright Nestor R. Gonzalez. Published with permission.

cavernous sinus to the bilateral inferior petrosal sinuses. There was also retrograde filling of a prominent right superior ophthalmic vein, which did not communicate with the facial vein.

\section{Operation}

The patient underwent multiple unsuccessful attempts at endovascular embolization. The first attempt at transvenous embolization, through the right inferior petrosal sinus, was aborted after multiple attempts to gain access to the fistula were unsuccessful. Although access to the cavernous sinus was straightforward, the microcatheter could not be advanced to the fistula itself. The second attempt at embolization was successful at transarterial occlusion
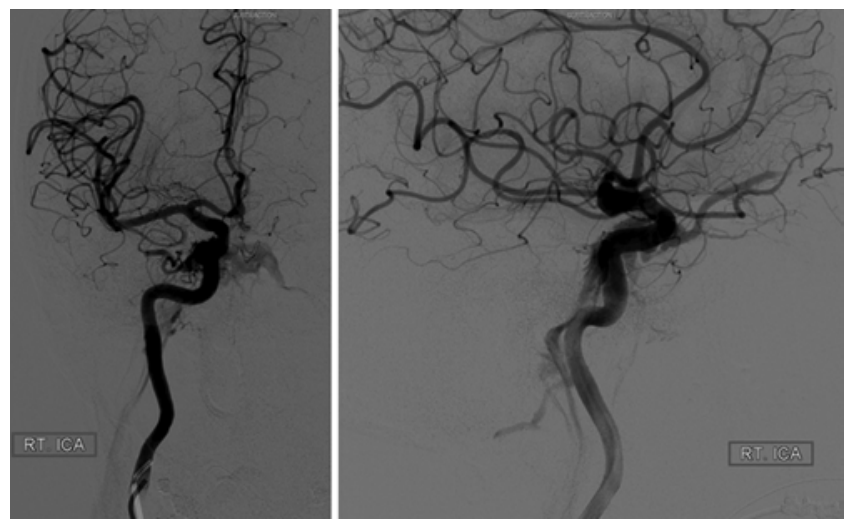

FIG. 2. Preoperative right ICA injection angiograms, anteroposterior (left) and lateral (right) views, showing the paracavernous venous plexus fistula. of the accessory meningeal artery, but this caused only minimal reduction in flow through the fistula. A repeated attempt at transvenous embolization, this time via bilateral inferior petrosal sinus approaches, was again unsuccessful because navigation of the microcatheter into the fistulous connection was not possible (Fig. 3). Navigation through the superior ophthalmic vein was considered, but it could not be accessed using endovascular techniques because it did not communicate directly with the facial vein and divided into smaller caliber veins at the angle of the orbit, making safe surgical exposure in the eyelid or direct puncture impossible.

Pursuit of an integrated open surgical and endovascular embolization of the fistula was chosen. High-resolution CT angiography (CTA) of the brain was conducted for preoperative planning and intraoperative navigation. The CTA images confirmed the location of the fistula sac lateral to the cavernous sinus in the paracavernous venous plexus. A lumbar drain was placed preoperatively to enhance brain relaxation. Through a right pterional craniotomy, an intradural, pretemporal approach to the lateral wall of the cavernous sinus was performed. Using the neuronavigation system, registered to a preoperative CTA image, the precise location of the fistula sac was identified and marked. Using femoral access, an intraoperative right common carotid artery angiogram was then acquired to map the features of the paracavernous fistula. Next, a spinal needle, registered to the neuronavigation system, was stereotactically guided into the paracavernous venous plexus. An angiogram was obtained through the spinal needle and it was confirmed that the tip was indeed in the fistula sac and that there was no filling of the internal carotid artery 

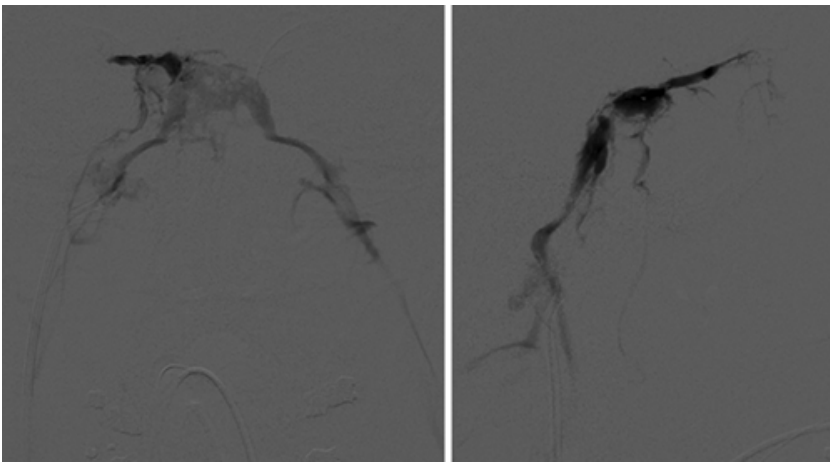

FIG. 3. Preoperative transvenous angiograms, anteroposterior (left) and lateral (right) views, showing the paracavernous venous plexus fistula.

(ICA) (Fig. 4). Next, 3 injections of ethylene vinyl alcohol (Onyx-18) were performed under fluoroscopic visualization. After the third injection of Onyx, an intraoperative right common carotid artery angiogram, through femoral access, confirmed the resolution of the fistula.

\section{Postoperative Course}

Postoperative noncontrast head $\mathrm{CT}$, in comparison with the preoperative CTA of the brain, showed the Onyx cast in the location of the fistula sac (Fig. 5). Immediately after surgery the patient had worsening of his chemosis, and an MRI of the brain revealed thrombosis of the inferior petrosal sinus. However, the patient's vision remained unchanged and he responded favorably to conservative treatment with supportive measures. He was discharged to home on postoperative Day 3. At the 3-month followup visit, the patient had complete resolution of his orbital chemosis and proptosis. Diagnostic cerebral angiography, at that time, showed no evidence of residual or recurrent fistula (Fig. 6).

\section{Discussion}

Some rare types of cavernous region DAVFs, involving the paracavernous venous plexus, can be mistaken for CCFs. In 2008, Shi et al. ${ }^{23}$ reported on 10 patients with spontaneous clival DAVFs treated by endovascular embo- lization. Given the close proximity and overlap of some of the arterial feeders and venous drainage, clival DAVFs can be misdiagnosed on cerebral angiography as indirect CCFs. However, they are anatomically distinct entities. The authors of this clinical study found on superselective angiograms that unlike CFFs, the fistula site for clival DAVFs is in the clival veins. While many types of CCFs are treated by transvenous approaches, ${ }^{9,10,14,15}$ Shi et al. ${ }^{23}$ concluded that the most effective treatment of clival DAVFs is transarterial embolization of the dural feeding arteries.

The literature also contains multiple reports of DAVFs involving the dura of the sphenoid bone that can mimic CCFs. ${ }^{1-3,7,8,18,22,24,27,29}$ This region of the paracavernous venous plexus contains multiple dural sinuses, and the nomenclature varies between authors. Detailed knowledge of the venous drainage of the superficial middle cerebral vein (SMCV) can help to better understand the complicated venous anatomy of this area. The outflow of the SMCV was described anatomically in 1999 by San Millán Ruiz et al. ${ }^{20}$ and angiographically in 2000 by Gailloud et al. ${ }^{4}$ They found that the SMCV had 3 different drainage patterns in increasing frequency: 1) a medial group that drained into the lateral portion of the cavernous sinus (this was the traditional route that was thought to be most common); 2) an intermediate group that drained into a laterocavernous sinus (LCS); and 3) a lateral group that drained into the paracavernous sinus on the floor of the middle cranial fossa.

Cavernous region DAVFs that involve venous outflow through the LCS can be mistaken for CCFs., ${ }^{41,13,20,21}$ In an anatomical study, San Millán Ruiz et al. ${ }^{20}$ described the LCS as a vascular channel between the two dural layers of the anterior third of the lateral wall of the cavernous sinus. In almost all cases this vascular channel was a continuation of the SMCV and it drained into the superior petrosal sinus, the pterygoid plexus or, least commonly, the posterior part of the cavernous sinus. The authors concluded that since, in most cases, there was no communication between the vascular channel and the cavernous sinus, the LCS should be considered an independent venous pathway running parallel to the cavernous sinus. Furthermore, they pointed out that this anatomical variant has important clinical implications because DAVFs with
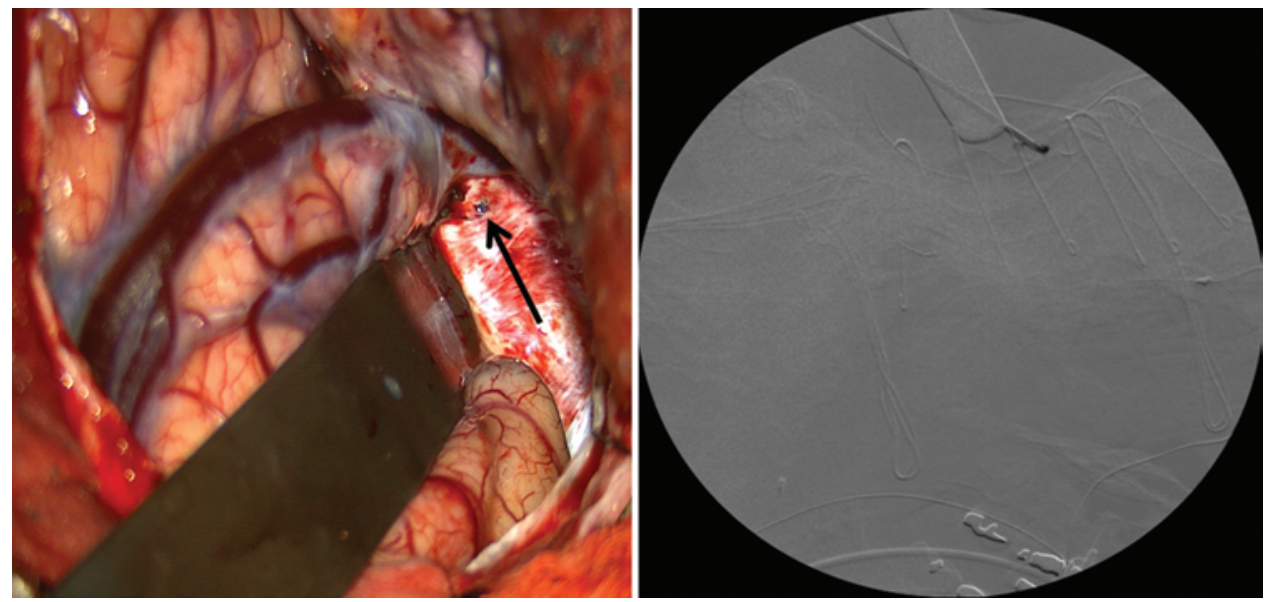

FIG. 4. The right intradural, pretemporal approach (left) with the injection site (arrow) in the lateral wall of the cavernous sinus. Intraoperative angiogram (right) demonstrating the tip of the spinal needle in the fistula sac. 

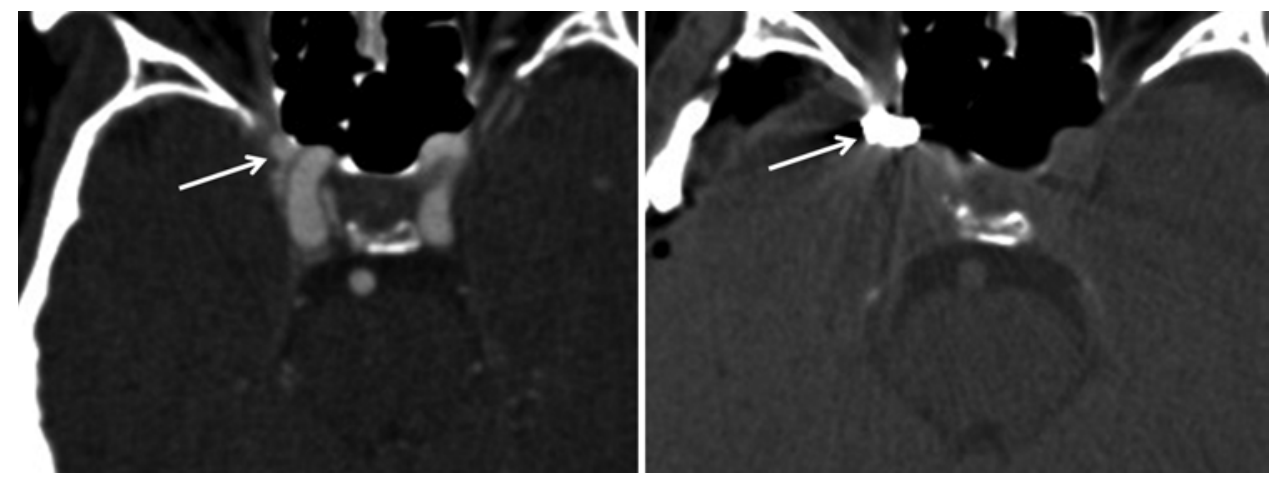

FIG. 5. Preoperative CTA image (left) showing the right paracavernous venous plexus fistula (arrow). Postoperative noncontrast CT scan (right) demonstrating the embolic material in the location of the fistula (arrow).

LCS drainage would not be accessible through standard endovascular routes, such as via the inferior petrosal sinus. San Millán Ruiz et al. ${ }^{21}$ went on, in 2007, to describe transvenous embolization of a DAVF of the LCS using a novel endovascular approach through the external jugular vein and the pterygoid plexus. In 2010, Lv et al. ${ }^{13}$ reported on a series of 7 patients with leptomeningeal vein (petrosal vein and SMCV) reflux secondary to cavernous region DAVFs with drainage via the LCS. Successful treatment was achieved in 4 patients with transarterial embolization and in 3 patients with transvenous approaches. Again, the authors concluded that recognition and detailed anatomical knowledge of the LCS is clinically important when planning the treatment of cavernous region DAVFs.

The most common venous drainage for the SMCV is the paracavernous sinus. This sinus is found within the dura of the greater sphenoid wing/floor of the middle cranial fossa. ${ }^{19,21,25,26}$ It is thought to be the embryological remnant of the tentorial sinus, described by Padget ${ }^{17}$ in 1956. Most authors describe the paracavernous sinus as the confluence of the sphenobasal and sphenopetrosal sinuses. ${ }^{4,19-21}$ The sphenobasal sinus and the sphenopetrosal sinus drain into the pterygoid plexus and the superior petrosal sinus, respectively. ${ }^{6}$

The dura of the lesser wing of the sphenoid bone contains a sinus known as the lesser wing of the sphe-
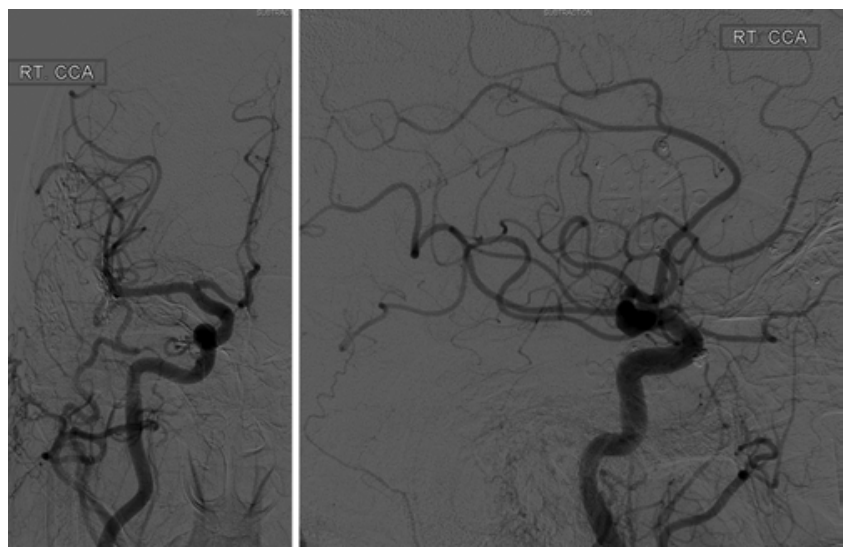

FIG. 6. Postoperative right ICA injection angiograms, anteroposterior (left) and lateral (right) views, showing resolution of the paracavernous venous plexus fistula. noid sinus (LWSS) and was described by Wolf et al. ${ }^{30}$ in 1963. A study by Kim et al. ${ }^{8}$ reported on the treatment of a DAVF involving the LWSS, which connects medially with the anterior and superior aspect of the cavernous sinus. This connection between the cavernous sinus and the LWSS can have an acute angle that makes transvenous approaches, especially from the inferior petrosal sinus, difficult. Therefore, the authors of this report recommended approaching these DAVFs through the facial vein.

Recently, Shi et al. ${ }^{22}$ published a report on a series of 11 patients with DAVFs within the dura of the sphenoid wing, 5 lesions involving the lesser wing of the sphenoid and 6 lesions involving the greater wing. Eight patients underwent transarterial embolization and 3 underwent transvenous embolization. The authors concluded that DAVFs of the greater sphenoid wing have a higher propensity for cortical venous drainage, and DAVFs of the lesser sphenoid wing can often mimic CCFs since they frequently drain into the cavernous sinus.

Open resection of DAVFs after incomplete endovascular embolization has been reported..$^{10,16,28}$ In the clinical study by Shi et al., ${ }^{22}$ of 11 DAVFs involving the sphenoid region, 2 required microsurgical disconnection of cortical draining veins after incomplete endovascular embolization. While this type of staged approach is well documented, reports of simultaneous integrated open surgery and endovascular embolization of CCFs are rare in the literature. Guerrero and colleagues ${ }^{5}$ and Krisht and Burson ${ }^{11}$ have published 2 technical case reports of a combined pretemporal-extradural approach for coil-assisted embolization of an indirect (Type D) CCF. In the first report, the authors used ultrasound guidance to help cannulate the cavernous sinus, while in the second report the lateral wall of the cavernous sinus was surgically dissected and exposed before coils were deployed using a cannula. In the current report, we approached the lateral wall of the cavernous sinus intradurally and used stereotactic neuronavigation to precisely locate the paracavernous venous plexus fistula before it was embolized with Onyx. The use of intraoperative neuronavigation combined with conventional transfemoral angiography and dynamic contrast-enhanced MR angiography for the surgical treatment of DAVFs has been previously reported..$^{28}$ As in the reports by Krisht and colleagues, the approach we are presenting also involved using an intra- 
operative femoral access angiogram to confirm that the fistula had been obliterated after embolization. Additionally, in our approach intraoperative fluoroscopy was used to perform an angiography through the spinal needle to confirm that the cannula was located in the fistula, and not the ICA, before embolization.

The anatomy of the paracavernous venous plexus is complex and the nomenclature is controversial. Consequently, since these rare types of DAVFs can mimic the much more commonly seen CCFs, a detailed understanding of the venous pathways is critically important in planning and executing their treatment. While some cavernous region DAVFs can still be completely embolized with endovascular treatment alone, other fistulas will remain open even after multiple endovascular approaches. It is these DAVFs that may require surgical treatment, and the current report presents an innovative integrated approach that combines stereotactic open surgery and endovascular embolization using Onyx through a surgical approach, which in contrast to other surgical techniques required only a needle puncture to occlude the sinus.

\section{Conclusions}

Paracavernous venous plexus fistulas are anatomically distinct from CCFs. Despite their proximity to the cavernous sinus, this anatomical difference may limit access to these lesions via an endovascular transvenous approach. Integrated stereotactically guided open surgical and intraoperative direct embolization is an effective treatment option.

\section{References}

1. Bitoh S, Arita N, Fujiwara M, Ozaki K, Nakao Y: Dural arteriovenous malformation near the left sphenoparietal sinus. Surg Neurol 13:345-349, 1980

2. Cohen JE, Gomori JM, Grigoriadis S, Spektor S, Rajz G: Dural arteriovenous fistula of the greater sphenoid wing region in neurofibromatosis type 1. Pediatr Neurosurg 44:172-175, 2008

3. Ezura M, Takahashi A, Mizoi K: Dural arteriovenous shunts involving the sphenoparietal sinus: a case report. Interv Neuroradiol 2:223-228, 1996

4. Gailloud P, San Millán Ruíz D, Muster M, Murphy KJ, Fasel $\mathrm{JH}$, Rüfenacht DA: Angiographic anatomy of the laterocavernous sinus. AJNR Am J Neuroradiol 21:1923-1929, 2000

5. Guerrero CA, Raja AI, Naranjo N, Krisht AF: Obliteration of carotid-cavernous fistulas using direct surgical and coilassisted embolization: technical case report. Neurosurgery 58:E382, 2006

6. Hacker H: Superficial supratentorial veins and dural sinuses, in Newton TH, Potts DG (eds): Radiology of the Skull and Brain. St. Louis: Mosby, 1974, Vol 2, pp 1851-1877

7. Khadavi NM, Mancini R, Nakra T, Tsirbas AC, Douglas RS, Goldberg RA, et al: Rare dural arteriovenous fistula of the lesser sphenoid wing sinus. Ophthal Plast Reconstr Surg 25:404-406, 2009

8. Kim MJ, Shin YS, Ihn YK, Kim BM, Yoon PH, Oh SY, et al: Transvenous embolization of cavernous and paracavernous dural arteriovenous fistula through the facial vein: report of 12 cases. Neurointervention 8:15-22, 2013

9. Kirsch M, Henkes H, Liebig T, Weber W, Esser J, Golik S, et al: Endovascular management of dural carotid-cavernous sinus fistulas in 141 patients. Neuroradiology 48:486-490, 2006

10. Klisch J, Huppertz HJ, Spetzger U, Hetzel A, Seeger W, Schumacher M: Transvenous treatment of carotid cavernous and dural arteriovenous fistulae: results for 31 patients and review of the literature. Neurosurgery 53:836-857, 2003

11. Krisht AF, Burson T: Combined pretemporal and endovascular approach to the cavernous sinus for the treatment of carotid-cavernous dural fistulae: technical case report. Neurosurgery 44:415-418, 1999

12. Lv X, Feng W, Li Y, Yang X, Jiang C, Liu L, et al: Cavernous region dural fistulas with venous drainage of laterocavernous sinus. Neurol India 59:190-194, 2011

13. Lv X, Jiang C, Li Y, Lv M, Wu Z: Endovascular treatment of dural fistulas with the venous outflow of laterocavernous sinus. Eur J Radiol 75:e129-e134, 2010

14. Meyers PM, Halbach VV, Dowd CF, Lempert TE, Malek AM, Phatouros CC, et al: Dural carotid cavernous fistula: definitive endovascular management and long-term followup. Am J Ophthalmol 134:85-92, 2002

15. Miller NR: Diagnosis and management of dural carotidcavernous sinus fistulas. Neurosurg Focus 23(5):E13, 2007

16. Natarajan SK, Ghodke B, Kim LJ, Hallam DK, Britz GW, Sekhar LN: Multimodality treatment of intracranial dural arteriovenous fistulas in the Onyx era: a single center experience. World Neurosurg 73:365-379, 2010

17. Padget DH: The cranial venous system in man in reference to development, adult configuration, and relation to the arteries. Am J Anat 98:307-355, 1956

18. Pakarinen S: Arteriovenous fistula between the middle meningeal artery and the sphenoparietal sinus. A case report. J Neurosurg 23:438-439, 1965

19. San Millán Ruíz D, Fasel JH, Rüfenacht DA, Gailloud P: The sphenoparietal sinus of breschet: does it exist? An anatomic study. AJNR Am J Neuroradiol 25:112-120, 2004

20. San Millán Ruiz D, Gailloud P, de Miquel Miquel MA, Muster M, Dolenc VV, Rufenacht DA, et al: Laterocavernous sinus. Anat Rec 254:7-12, 1999

21. San Millán Ruíz D, Oka M, Fasel JH, Clatterbuck R, Gailloud P, Murphy K: Transvenous embolization of a dural arteriovenous fistula of the laterocavernous sinus through the pterygoid plexus. Neuroradiology 49:665-668, 2007

22. Shi ZS, Ziegler J, Feng L, Gonzalez NR, Tateshima S, Jahan $\mathrm{R}$, et al: Middle cranial fossa sphenoidal region dural arteriovenous fistulas: anatomic and treatment considerations. AJNR Am J Neuroradiol 34:373-380, 2013

23. Shi ZS, Ziegler J, Gonzalez NR, Feng L, Tateshima S, Jahan $\mathrm{R}$, et al: Transarterial embolization of clival dural arteriovenous fistulae using liquid embolic agents. Neurosurgery 62:408-415, 2008

24. Smith JE, Epps J, Press HC Jr, Adair LB: Traumatic arteriovenous fistula between the middle meningeal artery and the sphenoparietal sinus: a case report and review of the world literature. J Natl Med Assoc 73:274-278, 1981

25. Suzuki Y, Matsumoto K: Variations of the superficial middle cerebral vein: classification using three-dimensional CT angiography. AJNR Am J Neuroradiol 21:932-938, 2000

26. Tanoue S, Kiyosue H, Okahara M, Sagara Y, Hori Y, Kashiwagi J, et al: Para-cavernous sinus venous structures: anatomic variations and pathologic conditions evaluated on fat-suppressed 3D fast gradient-echo MR images. AJNR Am J Neuroradiol 27:1083-1089, 2006

27. Unterhofer C, Chemelli A, Waldenberger P, Bauer R, Ortler $\mathrm{M}$ : Traumatic fistula between the middle meningeal artery and the sphenoparietal sinus. Acta Neurochir (Wien) 151:1301-1304, 2009

28. Vougioukas VI, Coulin CJ, Shah M, Berlis A, Hubbe U, Van Velthoven V: Benefits and limitations of image guidance in 
the surgical treatment of intracranial dural arteriovenous fistulas. Acta Neurochir (Wien) 148:145-153, 2006

29. Watanabe T, Matsumaru Y, Sonobe M, Asahi T, Onitsuka K, Sugita K, et al: Multiple dural arteriovenous fistulae involving the cavernous and sphenoparietal sinuses. Neuroradiology 42:771-774, 2000

30. Wolf BS, Huang YP, Newman CM: The superficial sylvian venous drainage system. Am J Roentgenol Radium Ther Nucl Med 89:398-410, 1963

\section{Author Contributions}

Conception and design: Gonzalez, Buchanan. Acquisition of data: all authors. Analysis and interpretation of data: Gonzalez, Dye. Drafting the article: Dye, Buchanan. Critically revising the article: all authors. Reviewed submitted version of manuscript: all authors. Approved the final version of the manuscript on behalf of all authors: Gonzalez. Administrative/technical/material support: Gonzalez. Study supervision: Gonzalez.

\section{Supplemental Information}

Proceedings

Portions of this work were presented in poster form at the Society of Neurointerventional Surgery International Endovascular Stroke Conference and Joint Cerebrovascular Section Annual Meeting, Honolulu, Hawaii, February 4, 2013.

\section{Correspondence}

Nestor Gonzalez, UCLA Department of Neurosurgery, 10833 LeConte Ave., Rm. 18-251 Semel, Los Angeles, CA 90095. email:ngonzalez@mednet.ucla.edu. 